\title{
Rapid and Mild Synthesis of Functionalized Naphthalenediimides
}

Ravuri S. K. Kishore, ${ }^{\dagger}$ Velayutham Ravikumar, ${ }^{\dagger}$ Gérald Bernardinelli, ${ }^{\dagger}$ Naomi Sakai, ${ }^{\dagger}$ and Stefan Matile*,†

${ }^{\dagger}$ Department of Organic Chemistry and ${ }^{\ddagger}$ Laboratory of X-ray Crystallography, University of Geneva, Geneva, Switzerland

*To whom correspondence should be addressed. E-mail: stefan.matile@chiorg.unige.ch

\section{Supplementary Information}

Table of Content

$\begin{array}{ll}\text { 1. Materials and methods } & \text { S2 }\end{array}$

2. Abbreviations $\quad \mathrm{S} 2$

$\begin{array}{lll}3 . & \text { Synthesis of } N, B r-N D I & \text { S3 }\end{array}$

4. Synthesis of $N, N$-NDI $3 \quad$ S5

5. Synthesis of $O, O$-NDI $9 \quad$ S5

$\begin{array}{ll}\text { 6. Crystallographic data } & \text { S8 }\end{array}$

$\begin{array}{ll}\text { 7. Supplementary figures } & \text { S9 }\end{array}$

8. Supplementary references S16 
1. Materials and methods. As in ref. S1, Supplementary Information. All reactions were performed under $\mathrm{N}_{2}$ or argon atmosphere. Column chromatography was carried out on silica gel 60 (40-63 $\mu \mathrm{m})$. Analytical (TLC) and preparative thin layer chromatography (PTLC) were performed in silica gel $60(0.2 \mathrm{~mm})$ and silica gel GF $(1 \mathrm{~mm})$, respectively. $[\alpha]^{20}$ values were recorded on a Polarimeter, melting points $(\mathrm{mp})$ on a heating table. IR spectra were recorded on a FT-IR spectrometer (ATR) and are reported as wavenumbers $v$ in $\mathrm{cm}^{-1}$ with band intensities indicated as $\mathrm{s}$ (strong), m (medium), w (weak). ESI-MS are reported as $\mathrm{m} / \mathrm{z}$ with relative intensities given in $\%$. HR ESI-MS on a QqTOF mass spectrometer. ${ }^{1} \mathrm{H}$ and ${ }^{13} \mathrm{C}$ spectra were recorded (as indicated) either on a $300 \mathrm{MHz}, 400 \mathrm{MHz}$ or $500 \mathrm{MHz}$ spectrometer and are reported as chemical shifts ( $\delta$ ) in ppm relative to TMS $(\delta=0)$. Spin multiplicities are reported as a singlet $(\mathrm{s})$, doublet $(\mathrm{d})$, triplet $(\mathrm{t})$, quartet (q) and quintet (quint) with coupling constants $(J)$ given in $\mathrm{Hz}$, or multiplet $(\mathrm{m})$. Broad peaks are marked as br. ${ }^{1} \mathrm{H}$ and ${ }^{13} \mathrm{C}$ resonances were assigned with the aid of additional information from 1D \& 2D NMR spectra (H,H-COSY, DEPT 135, HSQC and HMBC). UV-Vis spectra were measured on a spectrophotometer and are reported as maximal absorption wavelength $\lambda$ in $\mathrm{nm}$ (extinction coefficient $\varepsilon$ in $\mathrm{mM}^{-1} \mathrm{~cm}^{-1}$ ). CD spectra were measured using a spectropolarimeter and are reported as wavelength $\lambda$ of the extremum in $\mathrm{nm}\left(\Delta \varepsilon\right.$ in $\left.\mathrm{M}^{-1} \mathrm{~cm}^{-1}\right)$.

2. Abbreviations. Alloc: Allyloxycarbonyl; Boc: $t$-Butoxycarbonyl; calcd: Calculated; Cbz: Benzyloxycarbonyl; DMF: N,N-Dimethylformamide; en: Ethylenediamine; EtOAc: Ethyl acetate; HRMS: High resolution mass spectrometry; Lys: L-Lysine; NDA: Naphthalenedianhydride; NDI: Naphthalenediimide; NTE: Naphthalenetetraester; rt: Room temperature. 


\section{Synthesis of $N, B r-N D I 11$}

2,6-Dibromonaphthalene-1,4,5,8-tetracarboxylic acid dianhydride (Br,Br-NDA) 10. This compound was obtained as a mixture with $B r$-NDA 14 and $B r_{4}$-NDA 15 following the procedure in reference $\mathrm{S} 2$ by treatment of commercially available naphthalene-1,4,5,8-tetracarboxylic dianhydride (12) with dibromoisocyanuric acid $(\mathbf{1 3})^{\mathrm{S} 3}$ in oleum $\left(20 \sim 25 \% \mathrm{SO}_{3}\right)$. As it was not possible to purify at this stage, we assumed quantitative formation of $\mathbf{1 0}$ and used for the following reactions. We have noticed batch-to-batch difference in the ratio of mono-, di-, and tetra-Br NDAs, which seemed to reflect the sensitivity of this reaction to the concentration of $\mathrm{SO}_{3}$ in oleum.

Alloc-en-[Br,Br]-NDI-Lys(Cbz)-NH2 16. $B r, B r$-NDA $10(1.0 \mathrm{~g}, 2.36 \mathrm{mmol})$ was suspended in acetic acid $(10 \mathrm{~mL})$ and heated to $80{ }^{\circ} \mathrm{C}$. To this suspension was added a mixture of Allocethylenediamine $5(0.702 \mathrm{~g}, 4.85 \mathrm{mmol})^{\mathrm{S} 1}$ and $\mathrm{H}-\mathrm{Lys}(\mathrm{Cbz})-\mathrm{NH}_{2} \mathbf{6}(1.18 \mathrm{~g}, 4.85 \mathrm{mmol})$ in acetic acid $(30 \mathrm{~mL})$ and heated for $15 \mathrm{~h}$ at $80{ }^{\circ} \mathrm{C}$. The reaction mixture was cooled to $\mathrm{rt}$ and diluted with EtOAc $(400 \mathrm{~mL})$. The organic layer was washed with $1 \mathrm{M} \mathrm{KHSO}_{4}\left(50 \mathrm{~mL}\right.$ x 2), $\mathrm{H}_{2} \mathrm{O}(50 \mathrm{~mL})$, brine $(50 \mathrm{ml})$ and finally dried over $\mathrm{Na}_{2} \mathrm{SO}_{4}$. Solvent was evaporated and the resultant residue was purified by column chromatography $\left(\mathrm{CH}_{2} \mathrm{Cl}_{2} / \mathrm{MeOH} 20: 1, R_{\mathrm{f}}=0.3\right)$ to yield $\mathbf{1 6}(0.23 \mathrm{~g}$, $12 \%$ from 12) as a pale yellow solid. mp: $182-183{ }^{\circ} \mathrm{C} ;[\alpha]^{20}{ }_{\mathrm{D}}-9.24\left(c 0.145, \mathrm{CHCl}_{3} / \mathrm{MeOH} 1: 1\right)$; IR: 3299 (m), 2936 (m), 1694 (s), 1658 (s), 1562 (s), 1423 (m), 1367 (s), 1210 (m); ${ }^{1} \mathrm{H}$ NMR (400 MHz, $\mathrm{CDCl}_{3} / \mathrm{CD}_{3} \mathrm{OD} 4: 1$, Fig. S1): $8.91(\mathrm{~s}, 2 \mathrm{H}), 7.29-7.21(\mathrm{~m}, 5 \mathrm{H}), 5.76-5.68(\mathrm{~m}, 1 \mathrm{H}), 5.59\left(\mathrm{dd},{ }^{3} J\right.$ $\left.(\mathrm{H}, \mathrm{H})=6.8 \mathrm{~Hz},{ }^{3} J(\mathrm{H}, \mathrm{H})=5.6 \mathrm{~Hz}, 1 \mathrm{H}\right), 5.14\left(\mathrm{~d},{ }^{3} J(\mathrm{H}, \mathrm{H})=17.2 \mathrm{~Hz}, 1 \mathrm{H}\right), 5.07\left(\mathrm{~d},{ }^{3} J(\mathrm{H}, \mathrm{H})=10.4\right.$ $\mathrm{Hz}, 1 \mathrm{H}), 4.92(\mathrm{~s}, 2 \mathrm{H}), 4.38\left(\mathrm{~d},{ }^{3} J(\mathrm{H}, \mathrm{H})=5.6 \mathrm{~Hz}, 2 \mathrm{H}\right), 3.52\left(\mathrm{t},{ }^{3} J(\mathrm{H}, \mathrm{H})=4.8 \mathrm{~Hz}, 2 \mathrm{H}\right), 3.07\left(\mathrm{t},{ }^{3} J\right.$ 
$(\mathrm{H}, \mathrm{H})=6.8 \mathrm{~Hz}, 2 \mathrm{H}), 2.26-2.22(\mathrm{~m}, 2 \mathrm{H}), 1.55-1.47(\mathrm{~m}, 2 \mathrm{H}), 1.37-1.19(\mathrm{~m}, 2 \mathrm{H}) ;{ }^{13} \mathrm{C}$ NMR $(125$ $\mathrm{MHz}, \mathrm{CDCl}_{3} / \mathrm{CD}_{3} \mathrm{OD}$ 4:1, Fig. S1): 180.8 (s), 172.4 (s), 161.5 (s), 161.3 (s), 1610 (s), 157.4 (s), 157.3 (s), 139.3 (d) 139.1(d), 136.7 (s), 132.9 (s), 128.6 (d, 2x), 128.4 (d), 128.2 (s), 128.1 (d, 2x), $128.0(\mathrm{~s}), 127.9(\mathrm{~d}), 125.5(\mathrm{~s}), 124.4(\mathrm{~s}), 117.4(\mathrm{t}), 66.6(\mathrm{t}), 65.6(\mathrm{t}), 55.4(\mathrm{~d}), 41.2(\mathrm{t}), 40.3(\mathrm{t}), 39.3$ (t), $29.5(\mathrm{t}), 27.9(\mathrm{t}), 23.7(\mathrm{t}) ; \mathrm{MS}\left(\mathrm{ESI}, \mathrm{CH}_{2} \mathrm{Cl}_{2}\right.$, largest of isotopic peaks): $814\left(100,[\mathrm{M}+\mathrm{H}]^{+}\right)$, 797 (23, $\left[\mathrm{M}-\mathrm{NH}_{2}\right]^{+}$; HR-MS (ESI, +ve, Fig. S3): Calcd for $\mathrm{C}_{34} \mathrm{H}_{31} \mathrm{Br}_{2} \mathrm{~N}_{5} \mathrm{O}_{9} \mathrm{Na}^{+}$: 834.0380, Found: 834.0443.

Alloc-en-[i-PrNH,Br]-NDI-Lys(Cbz)-NH2 11. Alloc-en-[Br,Br]-NDI-Lys(Cbz)-NH 16 (0.14 $\mathrm{g}, 0.172 \mathrm{mmol})$ was dissolved in stirred isopropylamine $(20 \mathrm{~mL})$. After $5 \mathrm{~min}$ at $\mathrm{rt}$, the red solution was evaporated to dryness under reduced pressure. Purification by column chromatography $\left(\mathrm{CH}_{2} \mathrm{Cl}_{2} / \mathrm{MeOH} 48: 2 ; R_{\mathrm{f}}=0.4\right)$ gave $11(0.123 \mathrm{~g}, 90 \%)$ as a red solid. mp: $111-112{ }^{\circ} \mathrm{C}$; UV/vis $\left(\mathrm{CH}_{2} \mathrm{Cl}_{2} / \mathrm{MeOH}\right.$ 1:1): 532 (3.1), 366 (3.7), 348 (3.4); $\mathrm{CD}\left(\mathrm{CH}_{2} \mathrm{Cl}_{2} / \mathrm{MeOH}\right.$ 1:1): 367 (+0.6), 348 (+0.5), 272 (+8.1); IR: 3341 (m), 1670 (s), 1637 (s), 1581 (s), 1443 (s), 1311 (s), 1215 (s), 1142 (s), 992 (m), 790 (m); ${ }^{1} \mathrm{H}$ NMR (400 MHz, $\mathrm{CDCl}_{3}, \mathrm{~N} / \mathrm{N}=$ regioisomeric equivalents, Fig. S2): 10.03/9.99 (d, $\left.{ }^{3} J(\mathrm{H}, \mathrm{H})=7.6 / 7.2 \mathrm{~Hz}, 1 \mathrm{H}\right), 8.75 / 8.67(\mathrm{~s}, 1 \mathrm{H}), 8.31 / 8.20(\mathrm{~s}, 1 \mathrm{H}), 7.33-7.31(\mathrm{~m}, 5 \mathrm{H})$, 6.37 (br s, 1H), $5.89-5.81(\mathrm{~m}, 1 \mathrm{H}), 5.73-5.68 / 5.68-5.64(\mathrm{~m}, 1 \mathrm{H}), 5.35-5.04(\mathrm{~m}, 2 \mathrm{H}), 5.04(\mathrm{~s}$, 2H), 4.88 (br s, 1H), $4.54-4.47$ (m, 2H), 4.26 - 4.18 (m, 3H), 3.53 (br s, 2H), 3.19 (br s, 2H), 2.41 - $2.22(\mathrm{~m}, 2 \mathrm{H}), 1.72($ br s, $2 \mathrm{H}), 1.62-1.59(\mathrm{~m}, 2 \mathrm{H}), 1.52 / 1.51\left(\mathrm{~d},{ }^{3} \mathrm{~J}(\mathrm{H}, \mathrm{H})=7.6 / 5.6 \mathrm{~Hz}, 6 \mathrm{H}\right) ;{ }^{13} \mathrm{C}$ NMR (125 MHz, $\mathrm{CDCl}_{3} / \mathrm{CD}_{3} \mathrm{OD}$ 6:1, N/N = regioisomeric equivalents, Fig. S2): 171.6/171.1 (s), $165.3(\mathrm{~s}), 162.0(\mathrm{~s}), 161.4 / 161.3(\mathrm{~s}), 156.5 / 156.4(\mathrm{~s}), 151.0 / 150.9$ (s), 138.5/138.2 (d), 136.5/136.4 (s), $132.8(\mathrm{~s}), 132.7$ (d), 128.7 (s), 128.4 (d, 2x), 128.0/127.9 (d, 2x), 126.9 (s), 122.9/122.8 (s), 
121.3/121.1 (d), $121.0(\mathrm{~s}), 120.5 / 120.2(\mathrm{~s}), 117.6(\mathrm{t}), 99.2(\mathrm{~s}), 66.5(\mathrm{t}), 65.9(\mathrm{t}), 55.1 / 54.2(\mathrm{~d}), 46.5$

(d), 45.1/45.0 (t), 40.6/40.5 (t), 39.6/39.4 (t), 29.6/29.5 (t), 28.2/28.0 (t), 23.7/23.6 (d), 23.2/23.1 (d), 23.0 (q, 2x); MS (ESI, +ve, largest of isotopic peaks): $793\left(100,[\mathrm{M}+\mathrm{H}]^{+}\right), 776(80,[\mathrm{M}-$ $\left.\mathrm{NH}_{2}\right]^{+}$); HR-MS (ESI, +ve): Calcd for $\mathrm{C}_{37} \mathrm{H}_{40} \mathrm{BrO}_{9} \mathrm{~N}_{6}{ }^{+}:$791.2034, Found: 791.1976.

\section{Synthesis of $N, N$-NDI 3}

Alloc-en-[i-PrNH,i-PrNH]-NDI-Lys(Cbz)-NH $\mathbf{N H}_{2}$. A solution of Alloc-en-[i-PrNH,Br]-NDILys $(\mathrm{Cbz})-\mathrm{NH}_{2} 11(8.5 \mathrm{mg}, 0.01 \mathrm{mmol})$ in isopropylamine $(1.5 \mathrm{~mL})$ was stirred in a sealed tube at $\mathrm{rt}$ for $48 \mathrm{~h}$. Reaction mixture was concentrated in vacuo and the resultant residue was purified by PTLC $\left(\mathrm{CH}_{2} \mathrm{Cl}_{2} / \mathrm{MeOH} 48: 2 ; R_{\mathrm{f}}=0.5\right.$ with $\left.\mathrm{CH}_{2} \mathrm{Cl}_{2} / \mathrm{MeOH} 47: 3\right)$ to give $3(4.2 \mathrm{mg}, 51 \%)$ as a blue solid. Analytical and spectroscopic data were as reported (Fig. S5). ${ }^{\text {S1 }}$

\section{Synthesis of $O, O$-NDI 9}

Tetraethyl 2,6-dibromonaphthalene-1,4,5,8-tetracarboxylate ( $\mathrm{Br}, \mathrm{Br}$-NTE) 17. $500 \mathrm{mg}$ of the $B r, B r$-NDA $10(1.2 \mathrm{mmol})$ was suspended in $5 \mathrm{~mL}$ of ethanol and $5 \mathrm{~mL}$ of ethyl iodide (62 mmol). To this was added $\mathrm{K}_{2} \mathrm{CO}_{3}(1 \mathrm{~g}, 7.0 \mathrm{mmol})$. The mixture was refluxed for $6 \mathrm{~h}$ by which time the yellow suspension turned grey. The reaction mixture was cooled, evaporated to dryness, redissolved in $\mathrm{CH}_{2} \mathrm{Cl}_{2}$ and washed with water. The organic layer was then dried over $\mathrm{Na}_{2} \mathrm{SO}_{4}$ and concentrated to give a grey solid. Purification by column chromatography $\left(\mathrm{CH}_{2} \mathrm{Cl}_{2} ; R_{\mathrm{f}}=0.4\right.$ with $\left.\mathrm{CH}_{2} \mathrm{Cl}_{2}\right)$ afforded $\mathrm{Br}, \mathrm{Br}$-NTE 17 (170 mg, 24\% from 12) as a colorless solid. mp: $143-144{ }^{\circ} \mathrm{C}$; IR: 
2980 (w), 1717 (s), 1559 (m), 1470 (m), 1444 (m), 1375 (m), 1303 (m), $1271(\mathrm{~s}), 1247$ (s), 1180 (s), 1166 (s), 1015 (s), 868 (m), 760 (m), 689 (m); ${ }^{1} \mathrm{H}$ NMR (400 MHz, CDCl 3 , Fig. S7): 8.08 (s, 2H), $4.39\left(\mathrm{q},{ }^{3} J(\mathrm{H}, \mathrm{H})=7.2 \mathrm{~Hz}, 4 \mathrm{H}\right), 4.36\left(\mathrm{q},{ }^{3} J(\mathrm{H}, \mathrm{H})=6.9 \mathrm{~Hz}, 4 \mathrm{H}\right), 1.44\left(\mathrm{t},{ }^{3} J(\mathrm{H}, \mathrm{H})=6.9 \mathrm{~Hz}, 6 \mathrm{H}\right)$,

$1.41\left(\mathrm{t},{ }^{3} \mathrm{~J}(\mathrm{H}, \mathrm{H})=7.2 \mathrm{~Hz}, 6 \mathrm{H}\right) ;{ }^{13} \mathrm{C}$ NMR (100 MHz, $\mathrm{CDCl}_{3}$, Fig. S7): 166.5 (s), 166.4 (s), 134.7 (s), $134.5(\mathrm{~s}), 132.9$ (s), 128.6 (s), $121.4(\mathrm{~s}), 62.5$ (d), 62.4 (d), 14.2 (t), 13.9 (t); MS (ESI, +ve): $592\left(90,\left[\mathrm{M}+\mathrm{NH}_{4}^{+}\right]\right), 529\left(95,[\mathrm{M}-\mathrm{OEt}]^{+}\right)$.

Tetraethyl 2,6-diethoxynaphthalene-1,4,5,8-tetracarboxylate $(O, O$-NTE $) 18$. To a freshly prepared solution of $\mathrm{NaOEt}(8 \mathrm{~mL}$ of $2 \mathrm{M}$ ) was added drop by drop a solution of $B r, B r-\mathrm{NTE} 17$ (240 mg, $0.417 \mathrm{mmol}$ ) in $3 \mathrm{~mL}$ of dry DMF. The resulting yellow suspension was heated at $50{ }^{\circ} \mathrm{C}$ for $4 \mathrm{~h}$. The reaction mixture was quenched with water and then evaporated to dryness in vacuo. The residue was re-dissolved in water and extracted with $\mathrm{CH}_{2} \mathrm{Cl}_{2}(3 \times 25 \mathrm{~mL})$. The organic layer was separated, dried over $\mathrm{Na}_{2} \mathrm{SO}_{4}$ and evaporated to give a yellow residue. Purification by column chromatography (petroleum ether/EtOAc 80:20; $R_{\mathrm{f}}=0.4$ with petroleum ether/EtOAc 80:20) gave $O, O$-NTE $18(95 \mathrm{mg}, 45 \%)$ as a yellow solid. mp: 180-181 ${ }^{\circ} \mathrm{C}$; IR: $2983(\mathrm{~m}), 1717(\mathrm{~s}), 1583(\mathrm{~m})$, $1341(\mathrm{~m}), 1216(\mathrm{~s}), 1179(\mathrm{~s}), 1156(\mathrm{~s}), 1026(\mathrm{~s}), 928(\mathrm{~m}), 879(\mathrm{~m}), 775(\mathrm{~m}), 696(\mathrm{~m}) ;{ }^{1} \mathrm{H}$ NMR (400 $\mathrm{MHz}, \mathrm{CDCl}_{3}$, Fig. S8): $7.65(\mathrm{~s}, 2 \mathrm{H}), 4.35\left(\mathrm{q},{ }^{3} J(\mathrm{H}, \mathrm{H})=7.2 \mathrm{~Hz}, 8 \mathrm{H}\right), 4.22\left(\mathrm{q},{ }^{3} J(\mathrm{H}, \mathrm{H})=6.9 \mathrm{~Hz}\right.$, $4 \mathrm{H}), 1.43\left(\mathrm{t},{ }^{3} J(\mathrm{H}, \mathrm{H})=6.9 \mathrm{~Hz}, 6 \mathrm{H}\right), 1.39\left(\mathrm{t},{ }^{3} J(\mathrm{H}, \mathrm{H})=7.2 \mathrm{~Hz}, 12 \mathrm{H}\right) ;{ }^{13} \mathrm{C} \mathrm{NMR}(100 \mathrm{MHz}$, $\mathrm{CDCl}_{3}$, Fig. S8): 167.9 (s), 166.9 (s), 153.4 (s), 133.2 (s), 125.2 (s) 120.5 (s), 119.5 (s) 66.2 (d), $61.9(\mathrm{~d}), 61.4(\mathrm{~d}), 15.1(\mathrm{t}), 14.3(\mathrm{t}), 14.2(\mathrm{t}) ; \mathrm{MS}(\mathrm{ESI},+\mathrm{ve}): 522\left(25,\left[\mathrm{M}+\mathrm{NH}_{4}{ }^{+}\right]\right), 505(50,[\mathrm{M}+$ $\left.\mathrm{H}]^{+}\right), 458\left(100,[\mathrm{M}-\mathrm{OEt}]^{+}\right)$; HR-MS (ESI, +ve): Calcd for $\mathrm{C}_{26} \mathrm{H}_{32} \mathrm{O}_{10} \mathrm{Na}^{+}:$527.1887, Found: 527.1898. 
Alloc-en-[EtO,EtO]-NDI-Lys(Cbz)-NH2 9. $O, O$-NTE $18(50 \mathrm{mg}, 0.13 \mathrm{mmol})$ was added to 10 $\mathrm{mL}$ of a $1 \mathrm{M}$ solution of $\mathrm{KOH}$ in isopropanol. The mixture was refluxed for $14 \mathrm{~h}$ and then evaporated to dryness. The obtained residue was dissolved in $5 \mathrm{~mL}$ of acetic acid giving a clear yellow solution. To this was added H-Lys $(\mathrm{Cbz})-\mathrm{NH}_{2} \mathbf{6}(20 \mathrm{mg}, 0.13 \mathrm{mmol})$ and 5 (40 $\mathrm{mg}, 0.13$ mmol) sequentially and the mixture was heated for $12 \mathrm{~h}$ at $80{ }^{\circ} \mathrm{C}$. H-Lys(Z)- $\mathrm{NH}_{2} 6(20 \mathrm{mg}, 0.127$ $\mathrm{mmol})$ and 5 (40 $\mathrm{mg}, 0.127 \mathrm{mmol}$ ) were added again to the reaction mixture and the mixture was heated further for $24 \mathrm{~h}$. The reaction mixture was then cooled to rt and diluted with EtOAc (100 $\mathrm{mL})$. The organic layer was washed with $1 \mathrm{M} \mathrm{KHSO}_{4}(25 \mathrm{~mL}$ x 2$), \mathrm{H}_{2} \mathrm{O}(25 \mathrm{~mL})$, brine $(25 \mathrm{~mL})$ and finally dried over $\mathrm{Na}_{2} \mathrm{SO}_{4}$. Solvent was evaporated and the resultant residue was purified by column chromatography $\left(\mathrm{CH}_{2} \mathrm{Cl}_{2} / \mathrm{MeOH} 97: 3 ; R_{\mathrm{f}}=0.5\right.$ with $\left.\mathrm{CH}_{2} \mathrm{Cl}_{2} / \mathrm{MeOH} 90: 10\right)$ affording 9 (23 $\mathrm{mg}, 24 \%)$ as a yellow solid. mp: $133-134{ }^{\circ} \mathrm{C} ;[\alpha]^{20}{ }_{\mathrm{D}}+4.29\left(c \quad 0.5, \mathrm{CH}_{2} \mathrm{Cl}_{2}\right) ; \mathrm{IR}: 3346(\mathrm{~m}), 2936$ (m), 1695 (s), 1654 (s), 1574 (s), 1446 (s), 1245 (s), 1209 (s), 1107 (m), 1024 (m), 792 (m), 737 (m); ${ }^{1} \mathrm{H}$ NMR (400 MHz, $\mathrm{CDCl}_{3}$, Fig. S9): 8.33 (s, 1H), 8.24 (s, 1H), 7.28 - $7.24(\mathrm{~m}, 5 \mathrm{H}), 5.73$ (m, $1 \mathrm{H}), 5.65\left(\mathrm{dd},{ }^{3} J(\mathrm{H}, \mathrm{H})=9.1 \mathrm{~Hz},{ }^{3} J(\mathrm{H}, \mathrm{H})=5.3 \mathrm{~Hz}, 1 \mathrm{H}\right), 5.20\left(\mathrm{~d},{ }^{3} J(\mathrm{H}, \mathrm{H})=16.9 \mathrm{~Hz}, 1 \mathrm{H}\right), 5.17(\mathrm{~m}$, $1 \mathrm{H}), 5.09\left(\mathrm{~d},{ }^{3} J(\mathrm{H}, \mathrm{H})=10.1 \mathrm{~Hz}, 1 \mathrm{H}\right), 4.96(\mathrm{~s}, 2 \mathrm{H}), 4.85\left(\mathrm{t},{ }^{3} J(\mathrm{H}, \mathrm{H})=5.1 \mathrm{~Hz}, 1 \mathrm{H}\right), 4.43(\mathrm{~s}, 2 \mathrm{H})$, $4.42\left(\mathrm{q},{ }^{3} J(\mathrm{H}, \mathrm{H})=7.0 \mathrm{~Hz}, 4 \mathrm{H}\right), 4.23($ br s, $2 \mathrm{H}), 3.49(\mathrm{~m}, 2 \mathrm{H}), 3.14(\mathrm{~m}, 2 \mathrm{H}), 2.38-2.16(\mathrm{~m}, 2 \mathrm{H})$, $1.63\left(\mathrm{t},{ }^{3} J(\mathrm{H}, \mathrm{H})=7.0 \mathrm{~Hz}, 6 \mathrm{H}\right), 1.58-1.48(\mathrm{~m}, 2 \mathrm{H}), 1.46-1.21(\mathrm{~m}, 2 \mathrm{H}) ;{ }^{13} \mathrm{C}$ NMR $(100 \mathrm{MHz}$, $\mathrm{CDCl}_{3}$, Fig. S9): 171.6 (s), 162.5 (s), 162.4 (s), 161.1 (s), 160.9 (s), 160.3 (s), 160.1 (s), 156.6 (s), $136.6(\mathrm{~s}), 132.9$ (d), 128.6 (d), 128.2 (d), 128.1 (d), 127.0 (s), 126.7 (s), 123.5 (s), 123.4 (s), 119.8 $(d), 119.7(d), 117.6(t), 110.5(s), 110.2(s), 66.7(t), 66.6(t), 65.6(t), 54.5(d), 40.6(t), 40.0(t)$, $39.8(\mathrm{t}), 29.6(\mathrm{t}), 28.2(\mathrm{t}), 23.8(\mathrm{t}), 14.9(\mathrm{q}) ; \mathrm{MS}(\mathrm{ESI},+\mathrm{ve}): 766\left(25,[\mathrm{M}+\mathrm{Na}]^{+}\right), 744(80,[\mathrm{M}+$ $\left.\mathrm{H}]^{+}\right), 727\left(100,\left[\mathrm{M}-\mathrm{NH}_{2}\right]^{+}\right)$; HR-MS (ESI, +ve): Calcd for $\mathrm{C}_{38} \mathrm{H}_{42} \mathrm{O}_{11} \mathrm{~N}_{5}{ }^{+}$: 744.2875, Found: 
744.2803.

\section{Crystallographic data}

$B r, B r$-NTE 17. $\mathrm{C}_{22} \mathrm{H}_{22} \mathrm{Br}_{2} \mathrm{O}_{8} ; M_{\mathrm{r}}=574.3$, Monoclinic, $P 2_{1} / n, a=9.6278(6), b=9.7598(8), c=$ 11.9405(7) $\AA ; \beta=90.372(7)^{\mathrm{o}} ; V=1121.97(13) \AA^{3} ; Z=2, \mu=3.66 \mathrm{~mm}^{-1}, d_{\mathrm{x}}=1.700 \mathrm{gcm}^{-3}, \mathrm{Mo}_{\mathrm{K}_{\alpha}}$ radiation $(\lambda=0.71073 \AA) ; 16321$ reflections measured at $150 \mathrm{~K}$ on a STOE IPDS diffractometer, 2713 unique reflections of which 1955 with $\left|F_{o}\right|>4 \sigma\left(F_{o}\right)$. Data were corrected for Lorentz and polarization effects and for absorption $\left(T_{\min }, T_{\max }=0.4630,0.7458\right)$. The structure was solved by direct methods (SIR97). ${ }^{\text {S4 }}$ All calculations were performed with the XTAL system. ${ }^{\text {S5 }}$ Full-matrix least-squares refinement based on $F$ using weights of $1 /\left(\sigma^{2}(F o)+0.00015\left(F o^{2}\right)\right)$ gave final values $R$ $=\omega R=0.024$ and $S=1.15(2)$ for 178 variables and 1999 contributing reflections.

CCDC 663914 contains the supplementary crystallographic data for this paper. These data can be obtained free of charge via www.ccdc.cam.ac.uk/conts/retrieving.html (or from the Cambridge Crystallographic Data Centre, 12 Union Road, Cambridge CB2 1EZ, UK; fax: (+ 44) 1223-336-033; or deposit@ccdc.cam.ac.uk). 
7. Supplementary figures
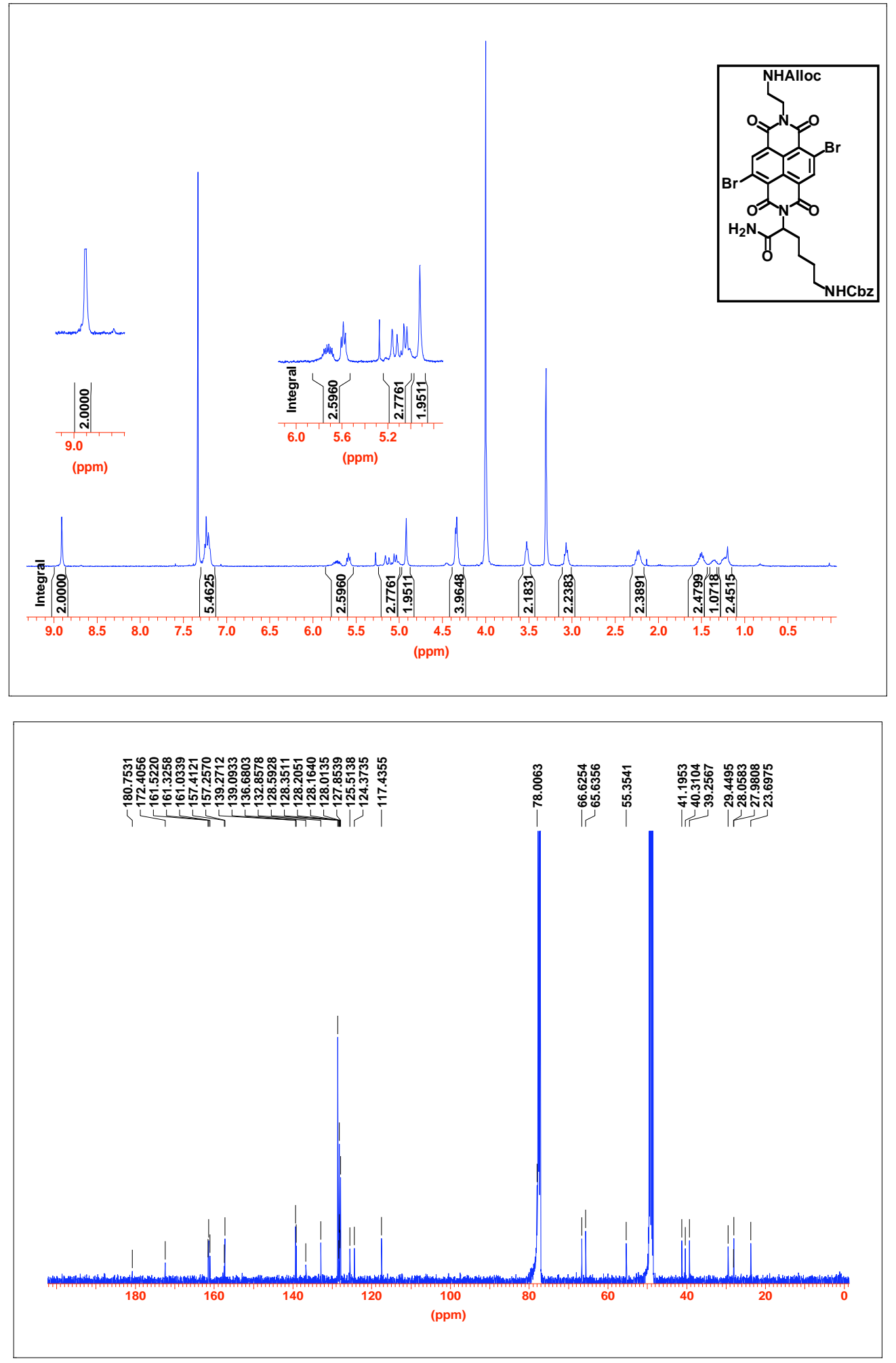

Figure S1. ${ }^{1} \mathrm{H}$ (top) and ${ }^{13} \mathrm{C}$ NMR (bottom) spectra of $\mathbf{1 6}$. 

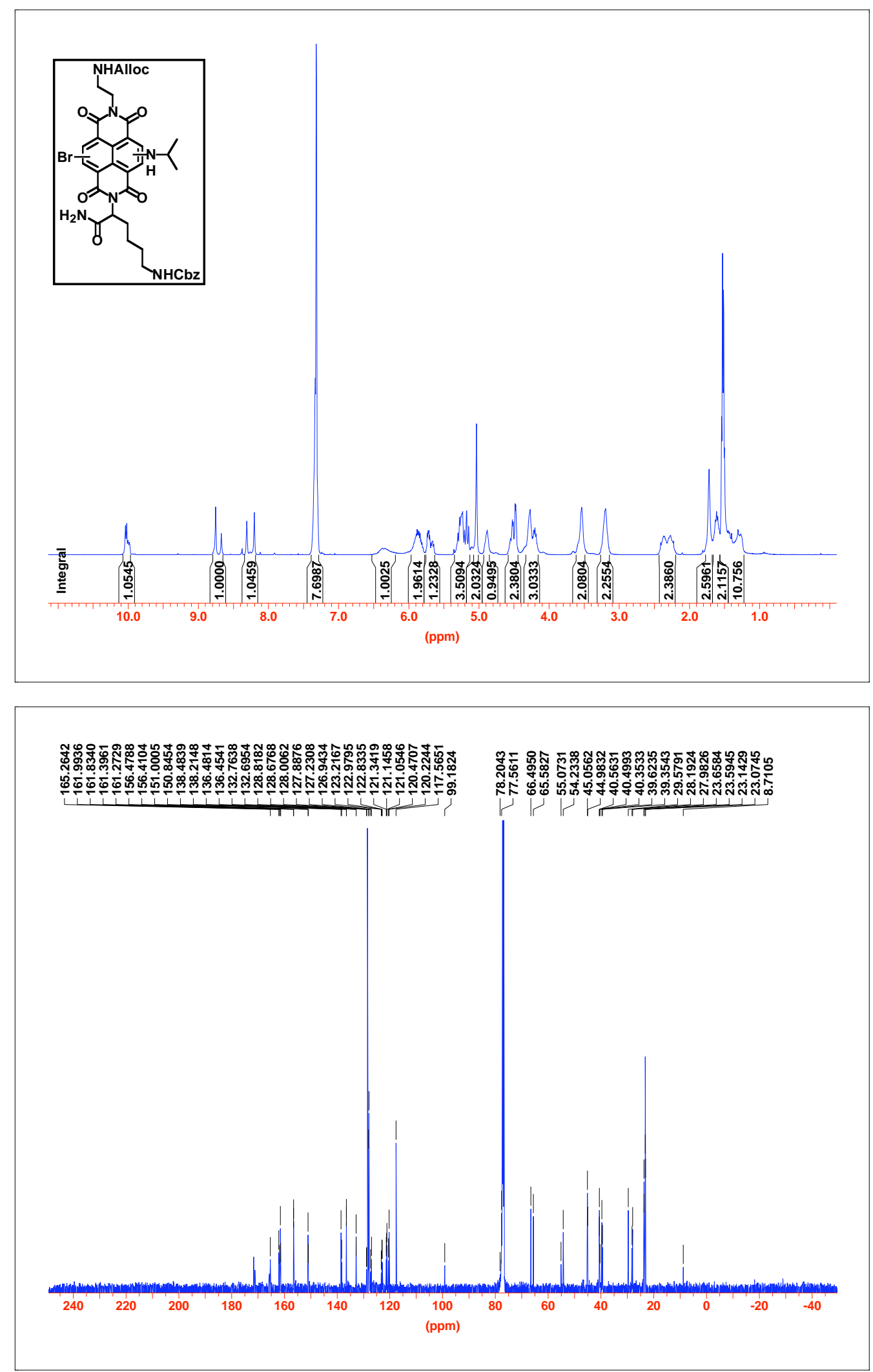

Figure S2. ${ }^{1} \mathrm{H}$ (top) and ${ }^{13} \mathrm{C}$ NMR (bottom) spectra of 11. 


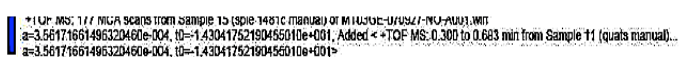

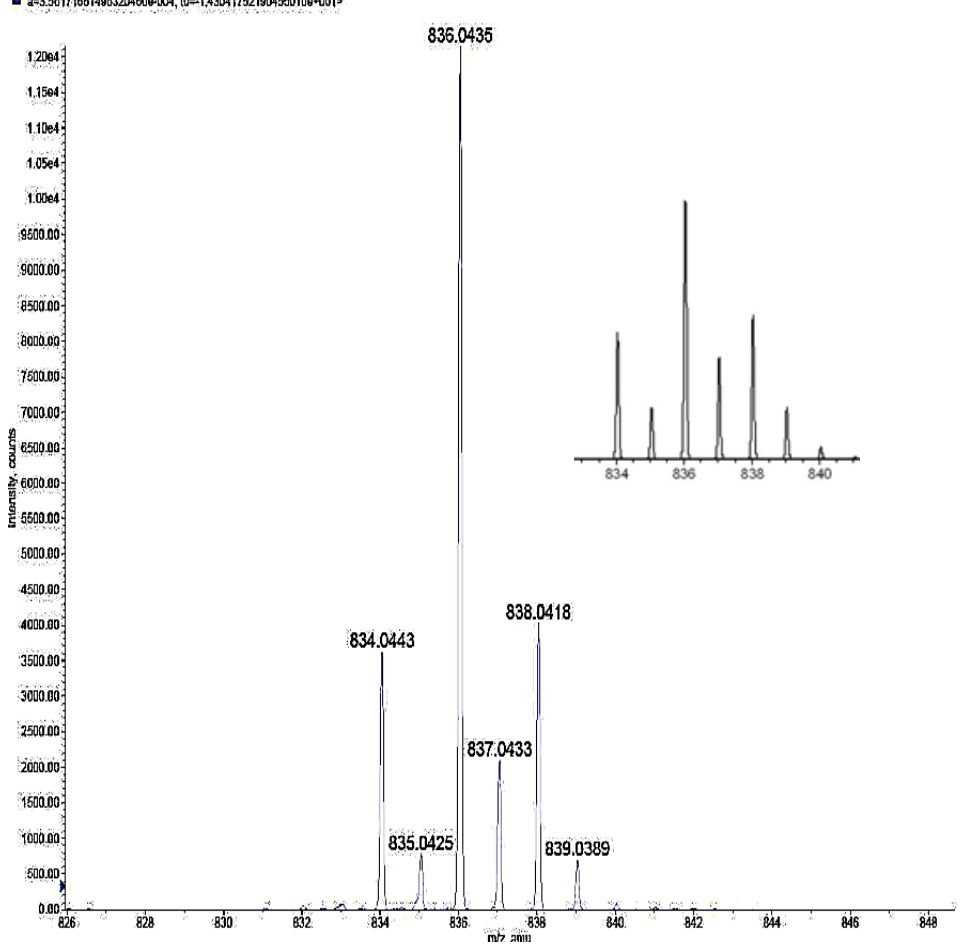

Figure S3. HR ESI-MS of 16; inset: simulated MS.

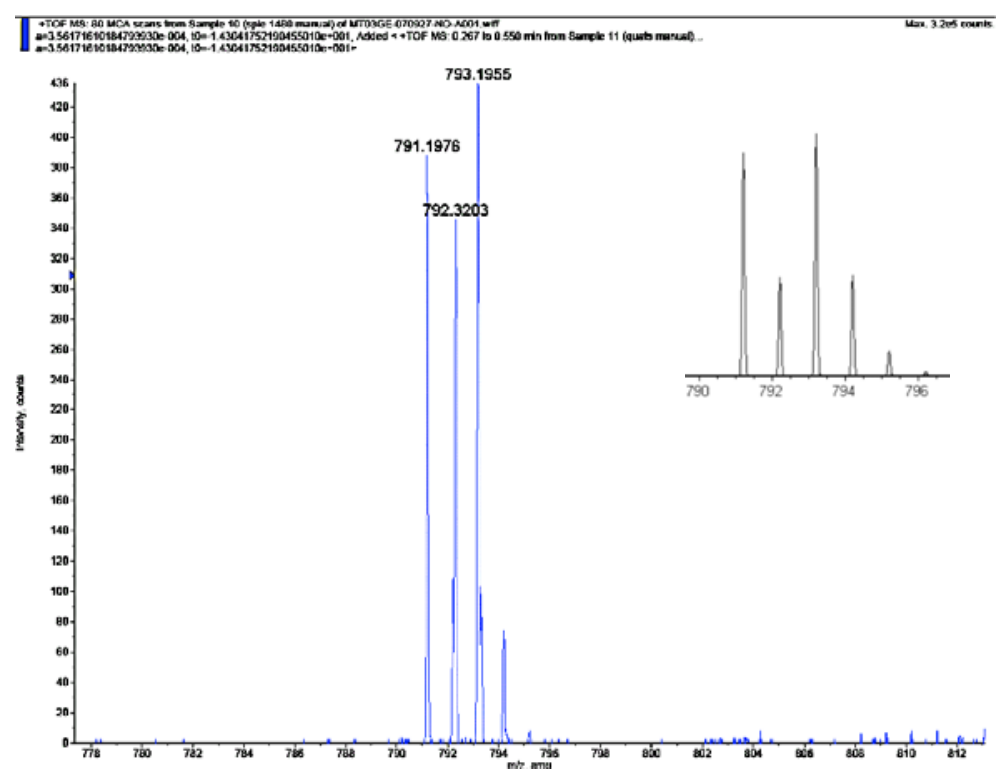

Figure S4. HR ESI-MS of 11; inset: simulated MS. 


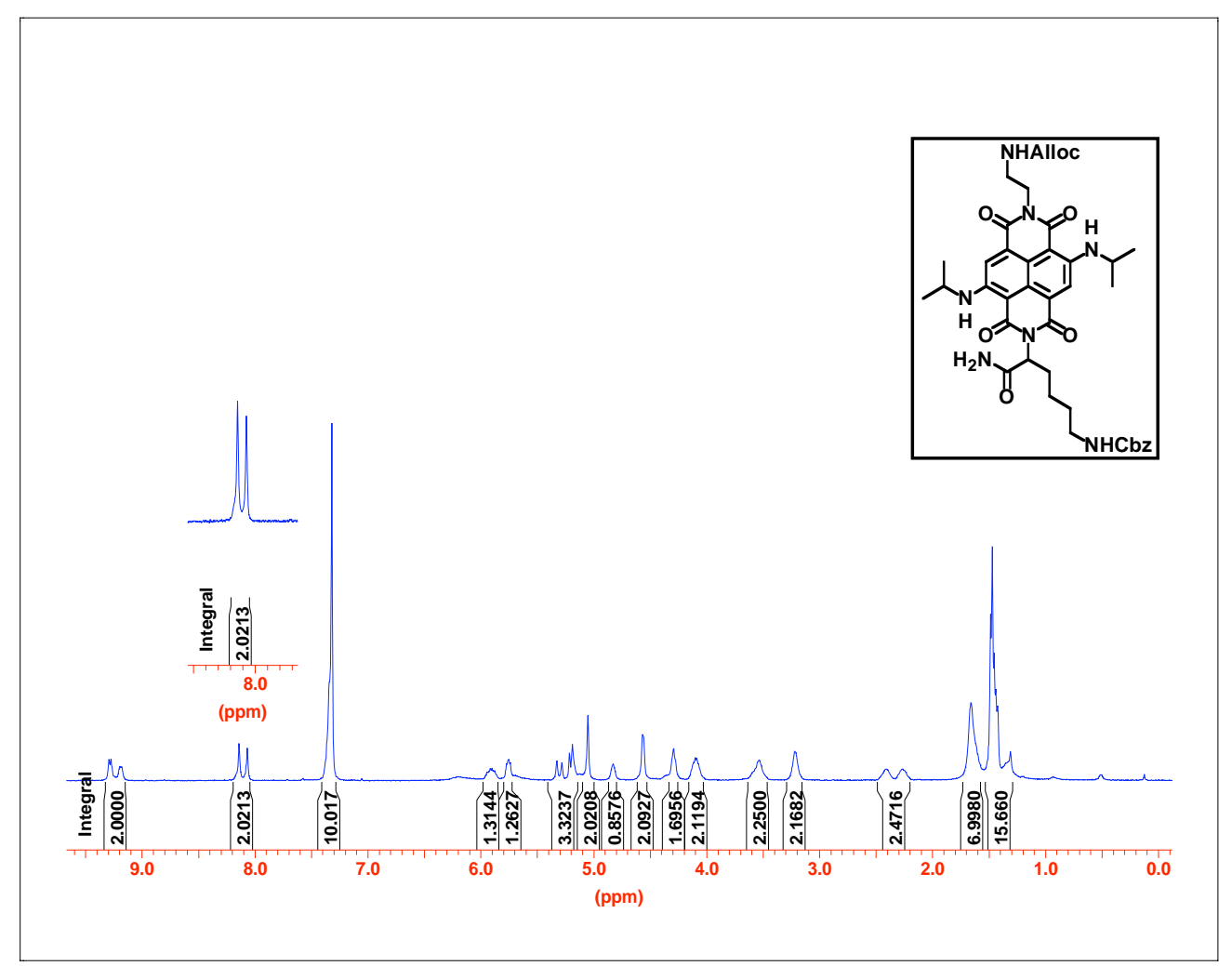

Figure S5. ${ }^{1} \mathrm{H}$ NMR spectrum of 3.

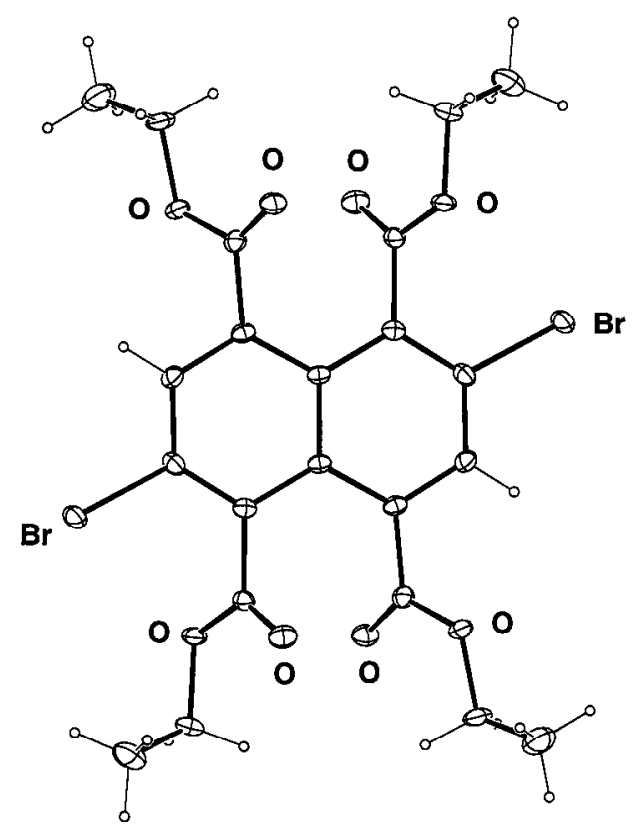

Figure S6. ORTEP view of compound 17. The molecule is located on a centre of inversion passing through the middle of the central bond. Ellipsoids are represented at the $50 \%$ probability level. 

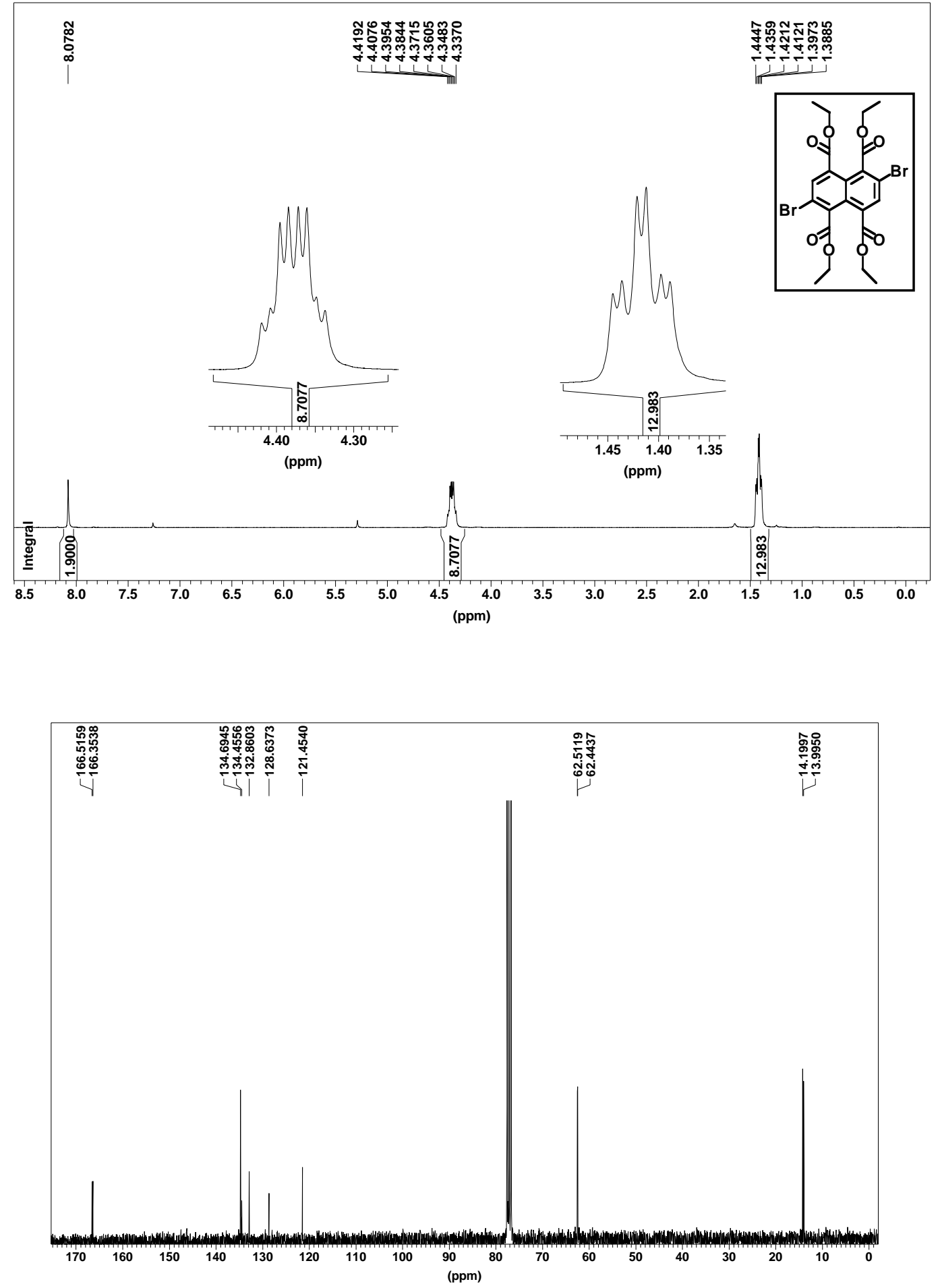

Figure S7. ${ }^{1} \mathrm{H}$ (top) and ${ }^{13} \mathrm{C}$ NMR (bottom) spectra of 17. 

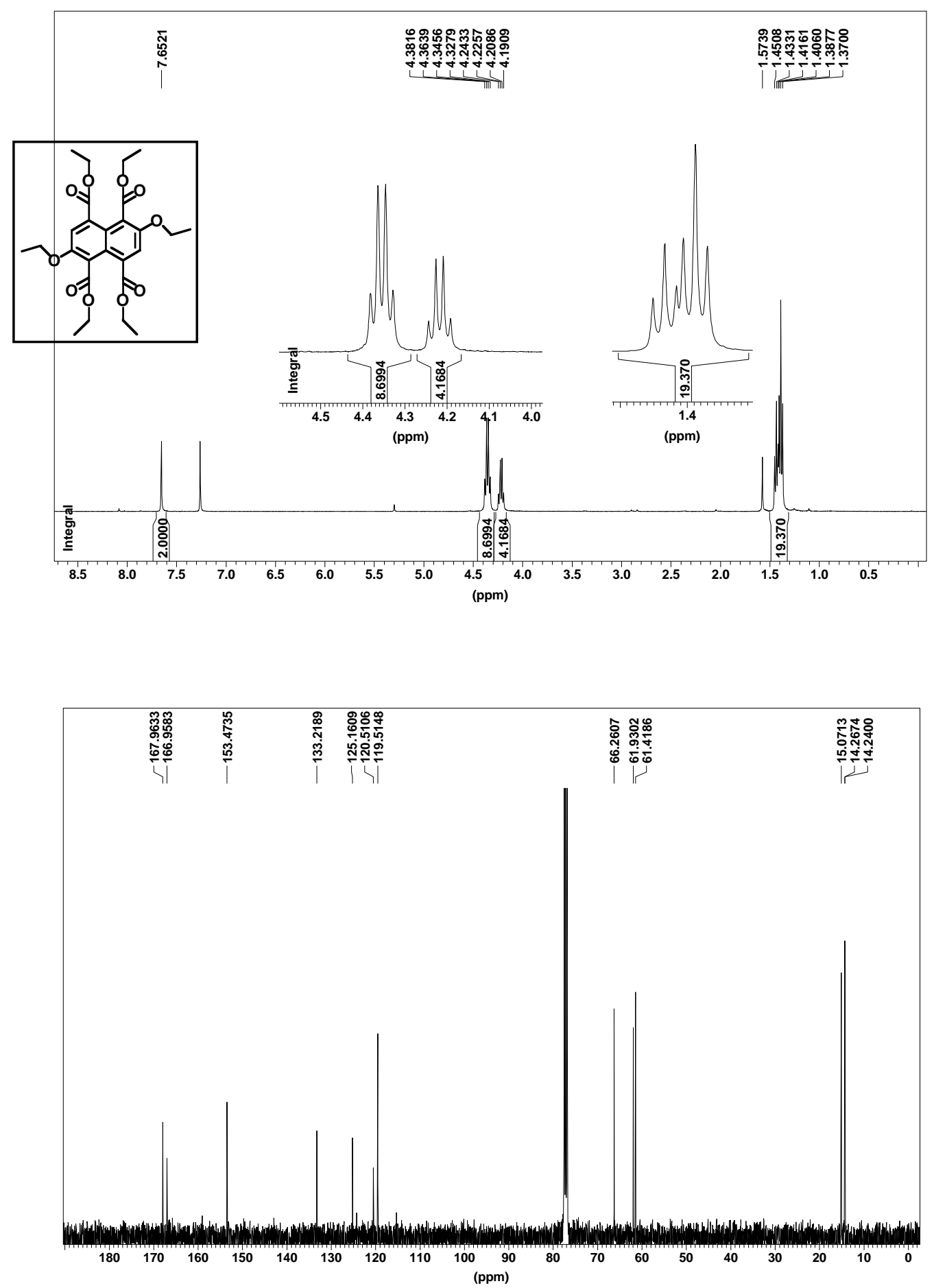

Figure S8. ${ }^{1} \mathrm{H}$ (top) and ${ }^{13} \mathrm{C}$ NMR (bottom) spectra of 18. 

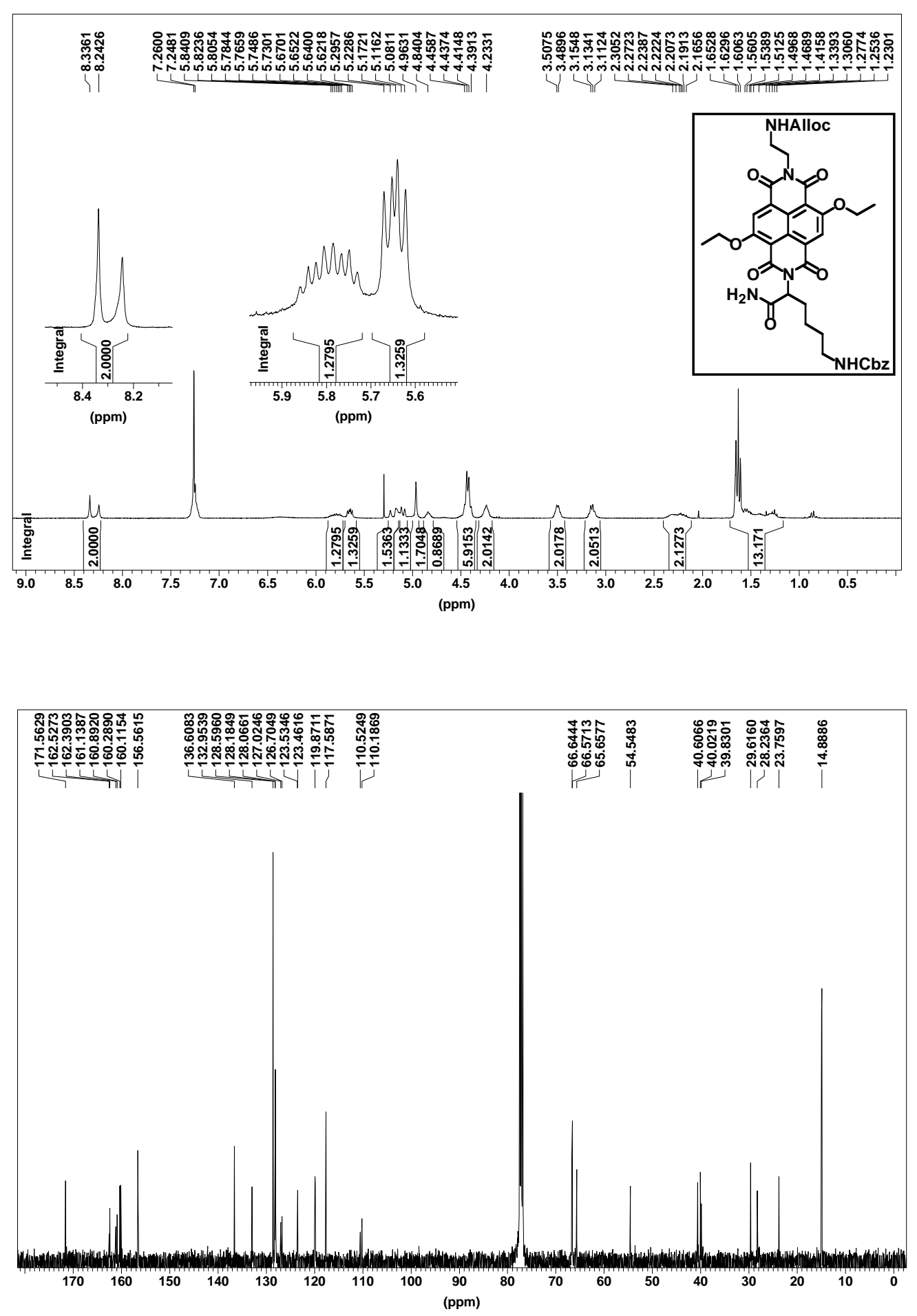

Figure S9. ${ }^{1} \mathrm{H}$ (top) and ${ }^{13} \mathrm{C}$ NMR (bottom) spectra of 9. 


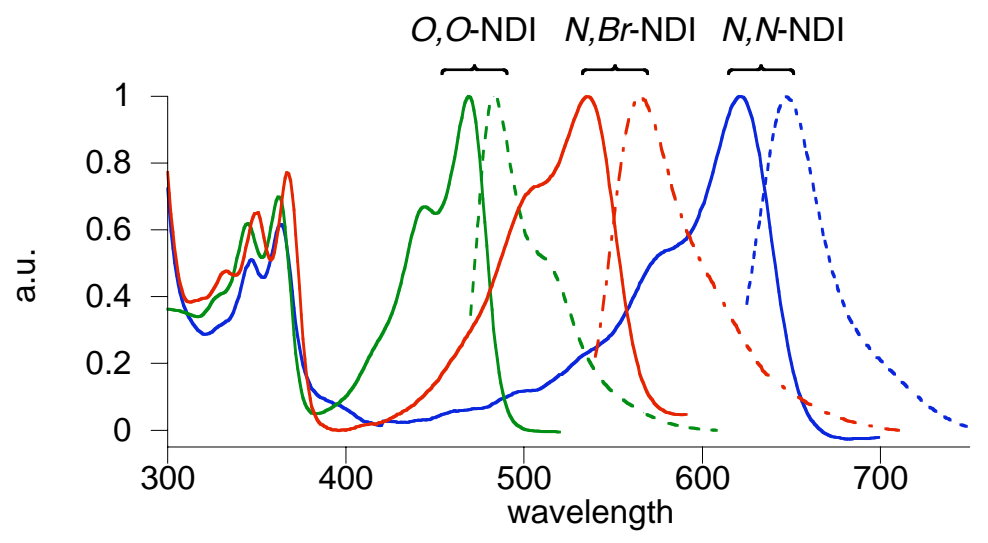

Figure S10. Intensity normalized absorption (solid) and fluorescence emission spectra (dashed) of $\mathbf{9}$ (green), $\mathbf{1 1}$ (red) and $\mathbf{3}$ (blue) in $\mathrm{MeOH}$.

\section{Supplementary references}

S1. $\quad$ Bhosale, S.; Sisson, A. L.; Talukdar, P.; Fürstenberg, A.; Banerji, N.; Vauthey, E.;

Bollot, G.; Mareda, J.; Röger, C.; Würthner, F.; Sakai, N.; Matile, S. Science 2006, 313, 84-86.

S2. $\quad$ Thalacker, C.; Röger, C.; Würthner, F. J. Org. Chem. 2006, 71, 8098-8105.

S3 Gottardi, W. Monatsh. Chem. 1968, 99, 815-822.

S4 Itomare, A.; Burla, M. C.; Camalli, M.; Cascarano, G.; Giacovazzo, C.; Guagliardi, A.; Moliterni, A. G. G.; Polidori, G.; Spagna, R. J. Appl. Cryst. 1999, 32, 115-119.

S5 Hall, S. R.; Flack, H. D.; Stewart, J. M. Xtal 3.2 User's Manual, Universities of Western Australia and Maryland, 1992. 